\title{
Levodopa induced ON-OFF motor fluctuations in Parkinson's disease related to rhythmical masticatory jaw movements
}

\author{
Stig Karlsson, Margaretha Persson, Bo Johnels
}

\begin{abstract}
The motor disturbance in Parkinson's disease affects all voluntary movement, including innate rhythmical processes such as gait, breathing, and chewing. While there are good descriptions and pathophysiological hypotheses of the changes in gait less is known about the way masticatory movements are affected. By means of a three-dimensional optoelectronic recording technique the differences were investigated in mandibular movement displacement, velocity, and masticatory cycle duration during levodopa induced OFF and ON states. Recordings were made before ordinary morning medication and one hour after medication with $200 \mathrm{mg}$ levodopa and a decarboxylase inhibitor (madopar). There were no differences before and after medication in the opening and closing duration of the masticatory cycle, but a significantly decreased occlusal level phase duration in the ON state was seen. Mandibular velocity and mandibular movement amplitude were significantly greater after medication. Thus the changes in dopamine transmission selectively influenced parts of the masticatory cycle but not the fundamental rhythmical pattern.
\end{abstract}

Parkinson's disease is caused mainly by a degenerative process in the dopaminergic neurons of the brain but changes in other transmitter systems may contribute. The most distinct cell reduction is found in the mesostriatal dopaminergic pathways. Due to functional and topographical differentiation various symptoms result when dopamine transmission drops in different parts of these pathways. Besides the well known cardinal signs of hypokinesia, muscle rigidity, and resting tremor there are often specific changes in body postural control, gait, and coordination of different parts of the body into purposeful acts. $^{12}$

When previously studying a simple motor task demanding the coordination of postural, locomotor, and manual body movements, Johnels $e t \mathrm{al}^{3}$ found a coordinative disintegration leading to a sequential rather than simultaneous performance of the partial movements of the act. Patients also often complain of difficulties in chewing and swallowing leading to loss of weight. This disturbance is thought to depend on hypokinesia but little is known of the mechanism behind the movement dis- turbances and its pathophysiology. Is it merely caused by the loss of motor speed (bradykinesia) or masticatory rhythmic changes or are there more complex disturbances? We made comparative observations of patients in levodopa induced $O N$ and $O F F$ states to study the mechanisms of the dopaminergic neurons' influence on rhythmical masticatory characteristics.

\section{Subjects and methods}

We studied 12 patients (mean age 65 years, range 45-76 years, five women and seven men) in different stages of Parkinson's disease. The degree of disability according to the five graded scale of Hoehn and Yahr ${ }^{4}$ varied from 2 to 4 . The duration of the disease showed great variation (mean 8 years, range 3-30 years). All patients gave informed consent.

Only individuals with no other disease were included in the study. Before being accepted, they were subjected to a clinical examination and a history of general diseases and drug administration was taken to ensure that there were no signs or symptoms of objective or subjective dysfunction of the masticatory system other than an altered motor function. The occlusion was stable, and there were no interferences in lateral or protrusive excursions of the mandible according to current definitions. The patients had been instructed to refrain from medication for 12 hours before the experiment.

Duplicate recordings of a masticatory sequence were made before and after medication with a single oral dose of levodopa (50-300 mg) combined with a decarboxylase inhibitor (madopar or sinemet). The second recording session was performed one hour after medication to obtain induced dopamine dependent recordings before (OFF state) and after levodopa medication (ON state). Oral motor function, expressed in terms of mandibular movement amplitude and velocity, was monitored with an optoelectronic method (Selspot AB, Partille, Sweden) which allowed for freedom of head movements. The threedimensional analysis system has been described in detail in previous reports. ${ }^{5-7}$ During chewing of peanuts, with the subject seated in a dental chair, duplicate recordings of masticatory sequences from start to swallowing were performed. The first masticatory cycle in each sequence was omitted because it was incomplete in many cases. In total 529 cycles were analysed in the OFF state and 488 in the ON state. 
For the purpose of analysis the masticatory cycle was defined as having three separate phases: mandibular opening (OP), mandibular closing (CP), and the occlusal level phase (OLP). OLP was defined as any position in which the mandible would be located from maximum intercuspation to a level $0.5 \mathrm{~mm}$ inferior to that position. OLP is also the transition phase between CP and OP. A quantitative analysis was performed of the following variables: the total cycle duration (TCD) and subdivisions of it (OP, CP, and OLP); the mean and maximum velocity in the $O P$ and $C P$ phases; and the mean three-dimensional mandibular spatial displacement in the $O P$ and $C P$ phases.

Student's $t$ test (two-tailed) was used to examine the significance of paired differences between the induced $O N$ and OFF levodopa states in the separate recordings. Pearson's product moment correlation test was used and the inter-relations between the variables were established by linear regression analysis.

\section{Results}

Masticatory cycle duration - In the quantitative analyses the total duration of a masticatory cycle was significantly $(p<0.05)$ shorter after levodopa medication (table 1 ). In addition, all phase durations decreased but only the change in the occlusal level phase (OLP) was significant. This change was significantly and positively correlated to performance before levodopa administration for both OLP $(r=0.66, \quad p<0.02)$ and $C P \quad(r=0.62$, $p<0.03)$. The analysis of the constituent phases in relation to total cycle duration (TCD) showed the CP to be significantly $(p<0.01)$ related in both the OFF and ON state.

Mandibular velocity-A significantly greater mean mandibular velocity was found for the OP $(p<0.01)$ and the CP $(p<0.001)$ in the ON state after levodopa (table 2). A similar,

Table 1 Masticatory cycle duration $(m s)$ in patients with Parkinson's disease in controlled OFF state and one hour after single dose of levodopa (ON state)

\begin{tabular}{llrllllll}
\hline & $O F F$ & & & & & & \\
\cline { 2 - 3 } & Mean & $S D$ & & Mean & $S D$ & p Value \\
\hline Opening phase & 137 & 60 & & 133 & 56 & NS \\
Closing phase & 215 & 98 & 201 & 77 & NS \\
Occlusal level phase & 146 & 91 & 115 & 67 & $<0.01$ \\
Total duration & 498 & 125 & 449 & 119 & $<0.05$ \\
\hline
\end{tabular}

^Significance for differences between OFF and ON state.

Table 2 Mean mandibular velocity $(\mathrm{mm} / \mathrm{s})$ in patients with Parkinson's disease at incisor point in opening and closing phases in controlled OFF ON state

\begin{tabular}{|c|c|c|c|c|c|}
\hline & \multicolumn{2}{|l|}{$O F F$} & \multicolumn{2}{|l|}{$O N$} & \multirow[b]{2}{*}{ p Value * } \\
\hline & Mean & $S D$ & Mean & $S D$ & \\
\hline $\begin{array}{l}\text { Opening phase (OP) } \\
\text { Closing phase (CP) } \\
\text { OP max } \\
\text { CP max }\end{array}$ & $\begin{array}{r}98 \\
71 \\
188 \\
136\end{array}$ & $\begin{array}{l}33 \\
18 \\
36 \\
30\end{array}$ & $\begin{array}{r}116 \\
86 \\
210 \\
161\end{array}$ & $\begin{array}{l}31 \\
19 \\
39 \\
33\end{array}$ & $\begin{array}{l}<0.01 \\
<0.001 \\
<0.01 \\
<0.001\end{array}$ \\
\hline
\end{tabular}

^Significance for differences between OFF and ON state. significantly increased maximum velocity for both OP and CP was recorded. Two of the patients did not increase their mandibular velocity after a single dose of levodopa. In addition, a clear positive correlation was found between before and after medication (OP; $r=0.91, p<0.0001$ and $C P ; \quad r=0.78$, $\mathrm{p}<0.0003)$.

Magnitude of mandibular displacement - The traversed three-dimensional spatial distance in vertical opening $(p<0.01)$ and closing was significantly $(p<0.001)$ increased at the second recording session after levodopa medication. This increase after medication was positively correlated for both the opening $(\mathrm{r}=0.70, \mathrm{p}<0.011)$ and the closing $(r=0.63, p<0.02)$ distances. No changes had occurred concerning the lateral component of the masticatory movements (Table 3). In patients with advanced disease the degree of ON-OFF fluctuations may vary strongly from time to time. In our patients, however, all improved, and only one preserved the same displacement as before medication.

Inter-relation between variables-A rhythmical masticatory pattern is characterised by cycle duration, mandibular velocity, and displacement. The cycle frequency is dependent on the inter-relation between displacement and velocity. A close linear relation was found when the

Table 3 Masticatory mandibular displacement $(\mathrm{mm})$ in patients with Parkinson's disease in controlled OFF state and one hour after single dose of levodopa (ON state)

\begin{tabular}{|c|c|c|c|c|c|}
\hline & \multicolumn{2}{|l|}{$O F F$} & \multicolumn{2}{|l|}{$O N$} & \multirow[b]{2}{*}{ p Value ${ }^{\star}$} \\
\hline & Mean & $S D$ & Mean & $S D$ & \\
\hline $\begin{array}{l}\text { Opening distance } \\
\text { Closing distance } \\
\text { Max lateral }\end{array}$ & $\begin{array}{r}10 \cdot 1 \\
9 \cdot 6 \\
4 \cdot 4\end{array}$ & $\begin{array}{l}2 \cdot 7 \\
2 \cdot 6 \\
1 \cdot 5\end{array}$ & $\begin{array}{r}12 \cdot 3 \\
11.9 \\
4.5\end{array}$ & $\begin{array}{l}3 \cdot 0 \\
2 \cdot 7 \\
1 \cdot 3\end{array}$ & $\begin{array}{l}<0.01 \\
<0.001 \\
\text { NS }\end{array}$ \\
\hline
\end{tabular}

^Significance for differences between OFF and ON state.
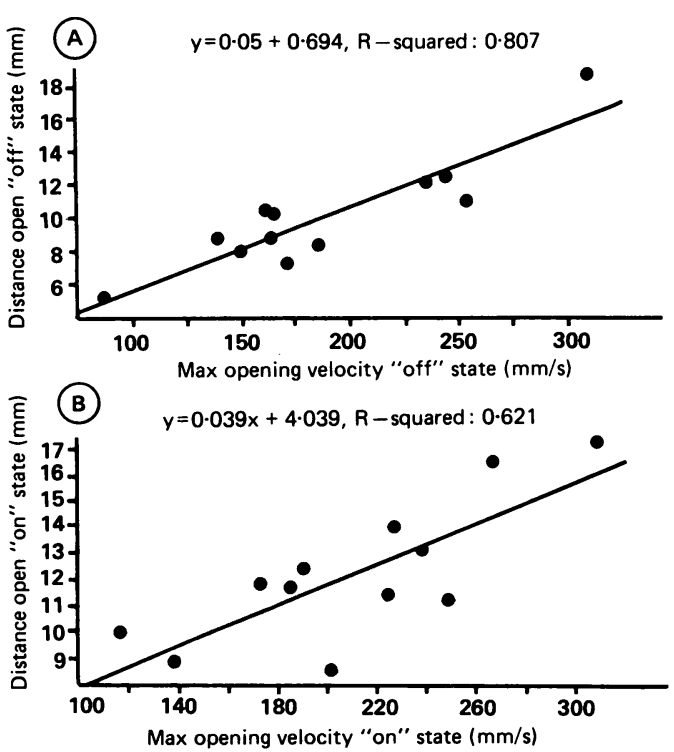

Figure 1 Scatter plots and linear relation between mandibular opening velocity and displacement before $(A)$ and after (B) single oral dose of levodopa. 
maximum opening velocity was plotted against vertical displacement for both the induced OFF and ON levodopa state (figure 1). The duration of the OP was independent and statistically unrelated to the amplitude of this movement (figure 2). Because of the mutual increase in velocity and displacement the duration of the OP remained unchanged. In the analysis of velocity and its relation to amplitude, maximum opening velocity was closely $(r=0.91)$ and significantly $(p<0.01)$ correlated to the vertical mandibular amplitude. Such a correlation was not detected for the closing velocity. The movement pattern was more irregular in the OFF state but stabilised after levodopa treatment (figure 3).

\section{Discussion}

There are several hypotheses concerning motor control of human mastication, resulting
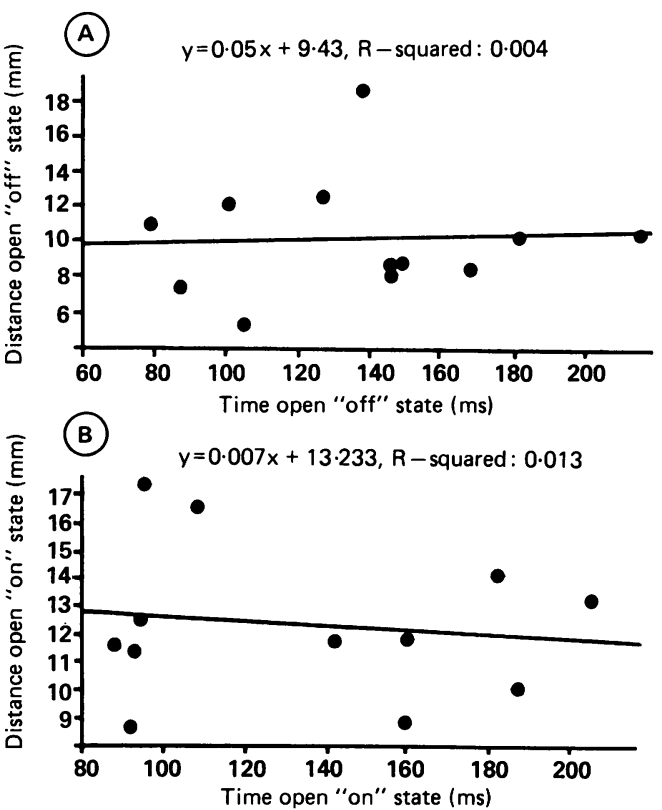

Figure 2 Scatter plots and linear relation between mandibular opening time and displacement before $(A)$ and after (B) single oral dose of levodopa.

\section{Jaw movement}
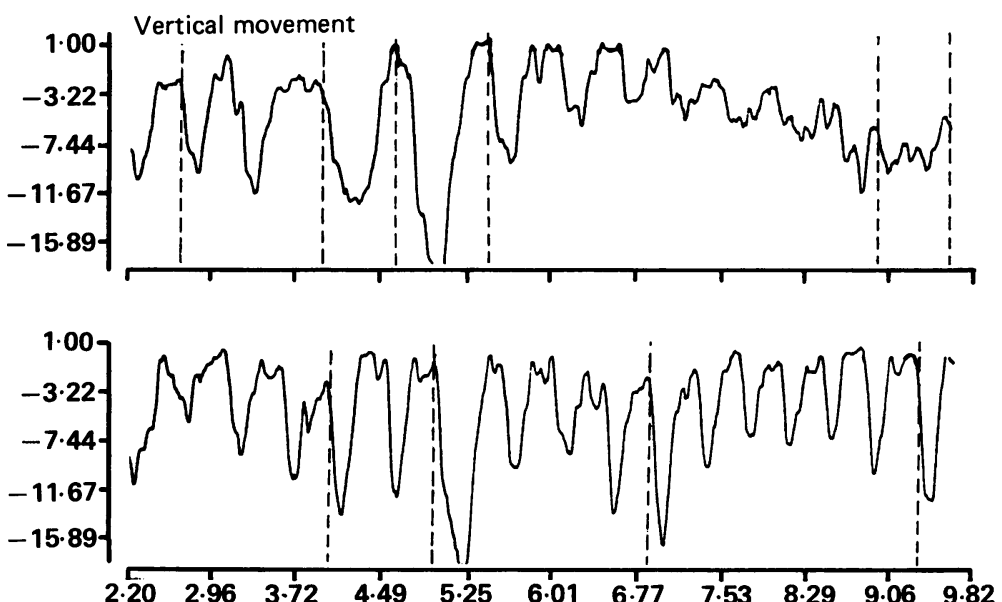

Figure 3 Examples of mandibular vertical displacement for one patient before (top) and after (bottom) levodopa medication. The units on the abscissa are time in seconds. The units on the ordinate are distance in $\mathrm{mm}$. from animal work. Some of these suggest a hierarchical organisation of neural networks containing specific programmes for automatised but voluntarily controlled functions such as chewing, breathing and swallowing, which require differentiated energy and coordinative motor output. Masticatory movements are generated in a bilaterally symmetric system, conditioned by preprogramming and reprogramming of neural networks after sensory information and updating through feedback loops. A central pattern generator (CPG) in the brainstem produces the basic output responsible for the masticatory rhythm by short latency polysynaptic pathways to jaw opening and closing motor neurons. ${ }^{8-15}$ This rhythmical output may provide an infrastructure on which modifications are superimposed and coordinated with functional requirements. The importance of dopaminergic neurons is unclear, but animal experiments have indicated that the CPG may be activated by dopamine but is not dependent on it for the generation of the basic pattern. ${ }^{16}$

Mastication, like walking, starts consciously and is continued in a cyclic repetitive pattern automatically performed until voluntarily turned off or when some kind of perturbation occurs. There is also evidence for similarities in the way rhythmical masticatory movements and gait are generated and temporo-spatially controlled. $^{17}$ In Parkinson's disease the gait pattern is often changed in the OFF situation and may be normalised by treatment with levodopa. ${ }^{18}$ This basal ganglia disorder may disturb the highly coordinated complex trigeminal neuromotor control of jaw movements in a similar way.

Many of the motor disturbances in this disease are caused by a general defect in the motor programme coordination system disrupting the normally smooth and simultaneous movments. ${ }^{219}$ Benecke et al found more improvement in the movement times of complex movements than in simple ones after levodopa treatment. ${ }^{20}$ There is also evidence for disturbed perioral reflexes in Parkinsonism, which suggests that basal ganglia pathways have a regulatory function and an impact on the sensorimotor control of oral motor function. $^{21}$

In our study we recorded the rhythmical masticatory motor performance of patients after taking levodopa (ON) and 12 hours after withdrawal (OFF) of normal medication to study the dopaminergic influence on the masticatory events and to observe similarities between the motor changes in chewing, gait, and limb movements. After treatment, the total cycle duration decreased $(p<0.05)$, but this was caused mainly by the duration or onset of the occlusal level phase (OLP) which significantly $(p<0.05)$ decreased after levodopa. The prolongation of OLP could be the effect of persisting masseter muscle activity or a delay in the onset of digastric (jaw opening) activation or both. As the mandibular closing phase (CP) time remained unchanged the latter is more likely. A salient feature was, however, that the other two phases, mandibular opening phase 
(OP) and $\mathrm{CP}$, remained constant irrespective of the increased mandibular opening amplitude and velocity. When the slopes of the regression lines for opening time, distance, and velocity were compared, only the two latter variables proved to be significantly correlated while OP time was unrelated.

Thus dopamine does not seem to influence the fundamental frequency drive of the masticatory movements. This might be because the CPG is discharging at a predetermined inherited or learned rhythm. An increased dopamine transmission, however, will probably improve the coordinative functions. As a result, the transition between the closing and opening phase will be facilitated and the duration of OLP reduced. ${ }^{22}$ In a previous report ${ }^{23}$ orofacial sensorimotor function in Parkinsonism was investigated and a deficit in the ability to utilise specific sensory inputs to guide and coordinate movements was found. The nigroneostriatal dopamine pathways seem consequently to be vital links in the control of oral motor behaviour.

In patients in the OFF state of advanced Parkinson's disease there is often difficulty in initiating movement. In our patients there was no problem in starting chewing on command, even though some of them exhibited clear difficulties in initiating walking. One patient had to count out loud to pace herself when initiating gait, and three patients had obvious problems in changing direction or passing through narrow passages when walking. Mastication was affected less than we expected in relation to the gait disorder. Although dependent on dopamine transmission, chewing and gait seem to depend on different coordinative mechanisms, one difference being the afferent stimulus evoked from a bolus of food in the mouth. Elicited feedback from oral mechanoreceptors will perhaps initiate and drive neural networks and motor programmes. Support for such differences comes from animal work. Lesions affecting the nigrostriatal pathways resulted in an increased perioral sensitivity and "abnormal biting reflexes" implying a role for the basal ganglia in oral sensorimotor regulation. ${ }^{2425}$

An impaired movement amplitude was recorded when the patients were OFF levodopa. Hence, the dopaminergic and nigrostriatal regulatory mechanism seems to be the same for jaw and limb movements and gait. ${ }^{2320}$ Bradykinesia was similarly affected and improved in the ON state. A decreased amplitude may be a part of a compensatory response and changed motor strategy to retain an optimised CPG rhythm.

In summary, the induced change in the dopamine transmission in patients with Parkinson's disease had an impact on modulation of coordinative CPG output and thereby affected the timing and energising trigeminal output to the jaw muscles. The basic features of the rhythmical masticatory pattern produced by neural networks in the brainstem was unchanged, however, and the CPG drive found to be independent of dopamine. These findings agree with results of animal experimental studies. ${ }^{16}$ The final processes responsible for modification of the CPG output generating the masticatory pattern and of related disturbances remain to be identified.

This investigation was supported by grants from the Royal Hvitfeldt Foundation, Handlande Svensson's Research Foundation, and the Swedish Dental Society. We thank Ms Aoneta Fougberg for her keen interest and skilful laboratory work.

1 Evarts EV, Teräväinen $H$, Calne DB. Reaction time in Parkinson's disease. Brain 1981;104:167-86.

2 Ingvarsson P, Johnels B, Steg G. Dissolution of moto program coordination in Parkinson's disease. In: Grillne S, Stein PSG, Forssberg H, Stuart DG, Herman RM, eds. Neurobiology of vertebrate locomotion. London: Macmillan, 1986;105-20.

3 Johnels B, Ingvarsson PE, Thorselius M, Valls M, Steg G. Disability profiles and objective quantitative assessment in Parkinson's disease. Acta Neurol Scand in Parkinson's

4 Hoehn MM, Yahr MD. Parkinsonism: onset, progression and mortality. Neurology (Cleveland) 1967;5:427-42.

5 Karlsson S. Recording of mandibular movements by intraorally placed light-emitting diodes. Acta Odontol Scand 1977;3:111-7

6 Karlsson S, Carlsson GE. Recording of masticatory mandibular movements and velocity by an optoelectronic method. Int I Prosthodont 1989;2:490-6.

7 Karlsson S, Carlsson GE. Characteristics of mandibular masticatory movement in young and elderly dentate subjects. F Dent Res 1990;69:473-6.

8 Hannam AG, Matthews B. Reflex jaw opening in response to stimulation of periodontal mechanoreceptors in the cat. Arch Oral Biol 1969;14:415-9.

9 Lund JP, Dellow PG. Rhythmic masticatory activity of hypoglossal motorneurons responding to an oral stimulus. Exp Neurol 1973;40:243-6.

10 Lund JP. Sensorimotor integration in the control of mastication. In: Klineberg I, Sessle BJ, eds. Orofacial pain and tion. In: Klineberg I, Sessle BJ, eds. Orofacial pain and Neuromuscular

11 Lund JP, Sasamoto K, Murakami T, Olsson KA. Analysis of rhythmical jaw movements produced by electrical stimulation of motor-sensory cortex of rabbits. $\mathcal{f}$ Neurophysiol 1984;52:1014-29.

12 Lund JP, Lamarre Y. Activity of neurons in the lower precentral cortex during voluntary and rhythmical jaw movements in the monkey. Exp Brain Res 1974 19:282-99.

13 Nakamura Y, Kubo Y. Masticatory rhythm in intracellular potential of trigeminal motorneurons induced by stimulation of orbital cortex and amygdala in cats. Brain Res 1978;148:504-9.

14 Sanes JN, Jennings UA. Centrally programmed patterns of muscle activity in voluntary motor behaviour of humans. Exp Brain Res 1984;54:23-30.

15 Enomoto S, Schwartz G, Lund JP. The effects of cortical ablation on mastication in the rabbit. Neurosc Lett ablation on mast

16 Chandler SH, Goldberg LJ. Differentiation of the neural pathways mediating cortically induced and dopaminergic activation of the control pattern generator (CPG) to rhythmical jaw movements in the anesthetized guinea pig. Brain Res 1984;323:297-300.

17 Bjursten LN, Norrsell K, Norrsell U. Behavioural repertoire of cats without cerebral cortex from infancy. Brain Res 1976;25:115-27.

18 Forssberg H, Johnels B, Steg G. Is Parkinsonian gait caused by regression to an immature walking pattern? Adv Neurol 1984;40:375-9.

19 Benecke R, Rothwell JC, Dick JPR, Day BL, Marsden CD. Performance of simultaneous movements in patients with Parkinson's disease. Brain 1986;109:739-57.

20 Benecke R, Rothwell JC, Dick JPR, Day BL, Marsden CD. Simple and complex movements off and on treatment in patients with Parkinson's disease. $\mathscr{f}$ Neurol Neurosurg patients with Parkinson's dise
Psychiatry 1987;50:296-303.

21 Caligiuri MP, Abbs JH. Response properties of the perioral reflex in Parkinson's disease. Exp Neurol reflex in $1987 ; 98: 563-72$.

22 Stern Y, Mayeux R Rosen J, Ilsen J. Perceptual motor dysfunction in Parkinson's disease: a deficit in sequential and predictive voluntary movement. $\mathcal{I}$ Neurol Neurosurg Psychiatry 1983;46:145-51.

23 Schneider JS, Diamond SG, Markham CH. Deficits in orofacial sensorimotor function in Parkinson's disease. Ann Neurol 1986;19:275-82.

24 Plesh O, Biship B, McCall W. Effect of gum hardness on chewing pattern. $\mathcal{f}$ Neurol 1986;92:502-12.

5 Nishino H, Ono T, Fukuda M, Sasaki K. Moneky substantia nigra (pars reticulata) neuron discharges during operant feeding. Brain Res 1985;334:190-3. 